\title{
Association of nucleophosmin/B23 with bladder cancer recurrence based on immunohistochemical assessment in clinical samples ${ }^{1}$
}

\author{
Ke-hung TSUI ${ }^{2}$, Horng-heng JUANG ${ }^{2}$, Tsong-hai $\mathrm{LEE}^{2}$, Phei-lang $\mathrm{CHANG}^{2}$, Chien-lun $\mathrm{CHEN}^{2}$, Benjamin Yat-ming \\ $\mathrm{YUNG}^{3,4}$ \\ ${ }^{2}$ Chang Gung Bioinformatics Center, Departments of Urology and ${ }^{3}$ Pharmacology, Chang Gung Memorial Hospital, Chang Gung University College of \\ Medicine, Kwei-Shan, Taiwan, China
}

\section{Key words}

bladder carcinoma recurrence; nucleophosmin/ B23; immunohistochemistry

${ }^{1}$ Project supported by Chang Gung Memorial Hospital (Grant No CMRPD1003-III, CMRPD32015, and CMRPG331193) and the National Science Council of Taiwan, China (Grant № NSC94-2745-B-182-001URD, NSC94-2320-B-182-009, and NSC 96-2314-B-182A-016-MY2).

${ }^{4}$ Correspondence to Prof Benjamin Yat-Ming YUNG.

$\mathrm{Phn} / \mathrm{Fax}$ 886-3-211-8615.

E-mail byung@mail.cgu.edu.tw

Received 2007-07-25

Accepted 2007-10-12

doi: $10.1111 / \mathrm{j} .1745-7254.2008 .00747 . \mathrm{x}$

\begin{abstract}
Aim: To investigate the possible correlation of nucleophosmin/B23 expression with bladder carcinoma recurrence. Methods: Surgically-resected bladder tumors staged pTa to pT4 were examined for nucleophosmin/B23 expression by immuno-histochemistry. The study group consisted of 132 consecutive patients surgically treated at Chang Gung Memorial Hospital between December 1998 and November 1999. The mean follow up was 72 months (range: 48-84 months). Results: Nuclear nucleophosmin/B23 staining was detected in $96 \%$ of advanced stage and poorly-differentiated tumors. Higher nucleophosmin/B23 levels were linked to more advanced tumor stages, grades, poor prognosis, and likelihood of recurrence $(P<0.05)$. The Cox multivariate analysis indicated the nucleophosmin/B23 expression as an independent indicator for tumor recurrence $(P=0.009)$. Conclusion: The results suggest that nucleophosmin/B23 is a favorable prognostic indicator for bladder cancer. Nucleophosmin/B23 could be a useful molecular tumor marker for predicting bladder cancer recurrence.
\end{abstract}

\section{Introduction}

Bladder cancer is the ninth most common cause of cancer for both sexes combined. The population-based, 5 -year survival rates range from $40 \%$ to $80 \%{ }^{[1]}$. Bladder cancer is also the second most common genitourinary malignant tumor in Taiwan. Approximately $70 \%$ of patients with bladder cancer are superficial and confined to the epithelium or subepithelial connective tissues at initial presentation. These groups of patients can be treated with transurethral resection with or without intravesical chemotherapy. Despite initial treatment, however, the recurrence rate for these tumors can be as high as $50 \%-70 \%$. In as many as $10 \%-35 \%$ of patients, disease progression invades muscles $^{[2]}$. Therefore, prompt diagnosis and treatment of disease recurrence and progression improve cancer control.

It has been suggested that the development and progression of bladder carcinoma result from the accumulation of multiple genetic mutations. Differing risks for bladder cancer are likely due to genetic predisposition that has been shown to modulate or neutralize carcinogen-derived bladder cancer ${ }^{[2]}$. The absence of risk stratification between aggressive and indolent $\mathrm{T} 1$, and carcinoma in situ CIS bladder cancer contributes to potentially excessive and frequent surveillance of patients with indolent or intermediate pathological features. There is presently no widelyaccepted, highly accurate, and non-invasive diagnostic test capable of predicting the probability of disease recurrence and progression. Currently, cystoscopy and urinary cytology are the gold standards for monitoring bladder cancer recurrence. However, cystoscopy and urinary cytology cannot predict clinical outcome. A highly accurate bladder tumor marker will likely prove extremely useful in the diagnosis and prognosis of patients with bladder cancer. Such a marker could avoid unnecessary cystoscopies in patients at low risk of disease recurrence and progression. Conversely, prediction of high risk of recurrence and progression in others could signify closer follow up.

The nucleophosmin/B23 gene has been shown to be involved in the regulation of cell growth, differentiation, and programmed cell death ${ }^{[3,4]}$. Nucleophosmin/B23 is sig- 
nificantly more abundant in tumor and proliferating cells than in normal resting cells. The overexpression of nucleophosmin/B23 in RNA and protein levels contributes to the pathogenesis of cancer ${ }^{[5]}$. The results from blocking the nucleophosmin/B23 expression by antisense oligonucleotides demonstrated that nucleophosmin/B23 is critical for increasing cancer cell resistance to induced differentiation and apoptosis ${ }^{[3,4]}$. The upregulation of nucleophosmin/B23 mRNA has recently been shown to be associated with bladder cancer recurrence and progression. The nucleophos$\mathrm{min} / \mathrm{B} 23$ gene may be associated with an increased risk of advanced tumor stage and recurrence ${ }^{[6]}$.

In the present study, we first focused on analyzing the relationship between the post-surgery recurrence rate for a mean of 65 months and the nucleophosmin/B23 mRNA expression. Next, we investigated by immunohistochemistry the nucleophosmin/B23 protein levels in bladder carcinoma and their correlation with clinicopathological variables. A survival analysis was also performed to define the prognostic relevance of the nucleophosmin/B23 protein expression.

\section{Materials and methods}

Patient population for tissue section This study received approval from the Institutional Review Board for the Protection of Human Subjects. A review of surgical specimens at Chang Gung Memorial Hospital (CGMH; Kwei-Shan, Tao-Yuan, Taiwan, China) between December 1998 and November 1999 yielded 132 cases of bladder carcinoma. The mean age of the patients was $58.7 \pm 8.6$ years (range, 43-78 years). The patients were followed postoperatively for a mean of 72 months (range, 48-84 months). Of the 132 cases, 99 were male (75\%) and 33 were female (25\%). The presence of cancer was confirmed histologically by the pathologist. Transitional cell carcinoma (TCC) was staged and graded according to the International Union Against Cancer $\mathrm{TNM}^{[7]}$ and World Health Organization classification ${ }^{[8]}$ after transurethral resection for superficial tumors and radical cystectomy for invasive tumors. The clinical background and history of these cases were reviewed (Table 1).

For the purpose of the comparison of survival rates, a matched group of 6 patients with Ta stage who underwent transurethral resection of bladder tumor was also selected and evaluated.

Immunohistochemistry The monoclonal antibody (MAb) to nucleophosmin/B23 was kindly provided by Dr PK CHAN (Department of Pharmacology, Baylor College of Medicine, Houston, TX, USA). The paraffin-embedded tissue blocks were sectioned in $2 \mathrm{~mm}$ slices and placed on
Table 1. Correlations between nucleophosmin/B23 immunohistochemistry staining and clinicopathological characteristics. ${ }^{\mathrm{b}} P<0.05$.

\begin{tabular}{|c|c|c|c|}
\hline \multirow[t]{2}{*}{ Variable } & \multicolumn{2}{|c|}{ Nucleophosmin/B23 staining } & \multirow[t]{2}{*}{$P$-value } \\
\hline & Low & High & \\
\hline Total $(n=132)$ & 79 & 53 & $0.09^{\mathrm{b}}$ \\
\hline \multicolumn{4}{|l|}{$\operatorname{Sex}(n=132)$} \\
\hline Male & 50 & 49 & \\
\hline Female & 16 & 17 & \\
\hline Age $(n=132)$ & & & $0.08^{\mathrm{b}}$ \\
\hline$<60$ & 38 & 29 & \\
\hline$>60$ & 28 & 37 & \\
\hline \multicolumn{4}{|l|}{ Histological grading } \\
\hline Grade 1 & 31 & 12 & \\
\hline Grade 2 & 25 & 28 & \\
\hline Grade 3 & 14 & 22 & $0.039^{\mathrm{b}}$ \\
\hline \multicolumn{4}{|l|}{ Tumor stage } \\
\hline $\mathrm{Ta}$ & 6 & 0 & \\
\hline $\mathrm{T} 1$ & 49 & 52 & \\
\hline $\mathrm{T} 2-\mathrm{T} 4$ & 8 & 17 & $0.049^{\mathrm{b}}$ \\
\hline \multicolumn{4}{|l|}{ Primary/Recurrent } \\
\hline Primary tumor & 43 & 31 & \\
\hline Recurrent tumor & 23 & 35 & $0.047^{\mathrm{b}}$ \\
\hline
\end{tabular}

We used a pTa tumor as a reference value to compare to the bladder tumor specimen for each immunohistochemistry stain (greater or less than reference value of pTa).

slides coated with poly- $L$-lysine. After overnight incubation in a $60{ }^{\circ} \mathrm{C}$ oven, the slides were dewaxed, rinsed in phosphate-buffered saline (PBS), and blocked for $5 \mathrm{~min}$ with $3 \%$ hydrogen peroxide to deprive the endogenous peroxidase activity. After antigen retrieval with the use of a microwave, the specimens were incubated with the antinucleophosmin/B23 MAb (diluted 1:100 in PBS) at $37{ }^{\circ} \mathrm{C}$ for $1 \mathrm{~h}$. The sections were then washed $4 \times 15 \mathrm{~min}$ in PBS and incubated with HRP/Fab polymer conjugate (PicTure ${ }^{\mathrm{TM}}$ bulk kit, Zymed USA) for $35 \mathrm{~min}$ at room temperature in a humid chamber. The sections were washed $4 \times 15 \mathrm{~min}$ with PBS and incubated with peroxidase substrate diaminobenzidine (Sigma, St Louis, MO, USA) for $5 \mathrm{~min}$. They were then counterstained with Mayer's hematoxylin for $2 \mathrm{~min}$, washed in running water for $5 \mathrm{~min}$, dehydrated with serial ethyl alcohol, and cleared with xylene. The slides were mounted in mounting medium ${ }^{[6,9]}$.

Assessment of nucleophosmin/B23 The extent and pattern of the nucleophosmin/B23-specific immunostaining within a tissue section was determined by the percentage of cells with a nucleus, cytoplasm, or cell membrane staining. Tumor sections were inspected at $\times 100$ and $\times 200$ magnifica- 
tion, and the overall percentage of cells on an immunostained section was determined according to the pattern of intracellular localization.

Statistical analysis For the statistical analysis, we utilized SPSS software system (SPSS for Windows, version 9.0; SPSS, Chicago, IL, USA). The Pearson $\chi^{2}$-test and Fisher's extract test were applied to assess the association of patients with relative tumor groups. The Student's t-test was employed to analyze associations with categorical clinicopathological variables. Survival and time to recurrence analyses were carried out by the Kaplan-Meier method, and the log-rank test was used to evaluate the prognosis for statistically significant differences among the groups. The Cox regression analysis was employed to identify statistically significant predictors of recurrence-free survival. Statistical significance was set at $P<0.05$.

\section{Results}

Association of nucleophosmin/B23 mRNA levels with high recurrence rates The association between nucleophosmin/B23 mRNA expression and tumor recurrence in bladder cancer was previously reported ${ }^{[6]}$. Methods based on RT-PCR had been used to detect bladder cancer in tumor specimens ${ }^{[12]}$. Thirty seven men and 13 women all had a transitional cell carcinoma of the bladder, including 34 primary and 16 recurrent bladder cancers. The overexpression of nucleophosmin/B23 mRNA was observed in 37.1\% of patients with stage pT1 and $73.3 \%$ of those with pT2-T4 carcinoma. An analysis of the nucleophosmin/B23 mRNA levels indicated that the upregulation of the nucelophos$\mathrm{min} / \mathrm{B} 23$ transcript may be an important prognostic factor for bladder cancer recurrence ${ }^{[6]}$. In the present study, we extended our previous expression study on the post-surgery recurrence rate to a mean of 65 months and examined nucleophosmin/B23 mRNA levels. The overexpression of nucleophosmin/B23 transcript was found to be significantly associated with a high recurrence rate of bladder cancer (log-rank statistic, $P=0.002$; Figure 1).

Immunohistochemical analysis Next, the protein expression of nucleophosmin/B23 was then assessed by immuno-histochemistry. Nucleophosmin/B23 staining of different intensities and percentages was found in the nucleoli, nuclei, and cytoplasm in varying degrees (Figure 2). As a shuttle protein involved in multiple cellular activity, the ubiquitous expression of nucleophosmin/B23 in active proliferated cancer cells is therefore expected. The overexpression was grossly overestimated or underestimated and its association with patient survival was assessed by the Cox survival analysis. Of the 132 cases evaluated, the

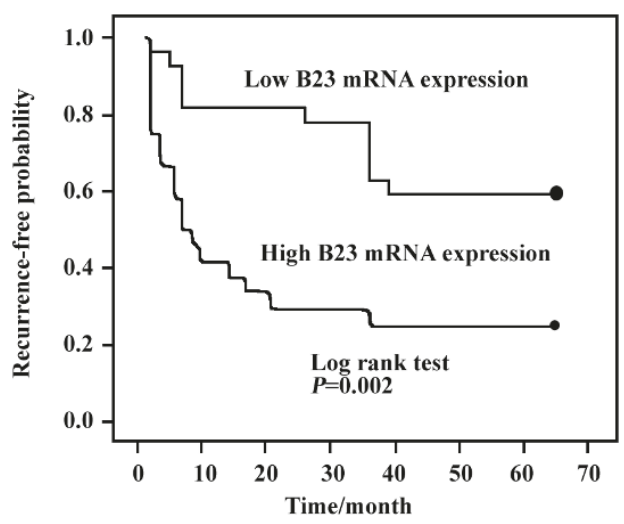

Figure 1. Kaplan-Meier survival analysis of necleophosmin/B23 mRNA expression and bladder cancer recurrence; differences assessed with logrank statistics.

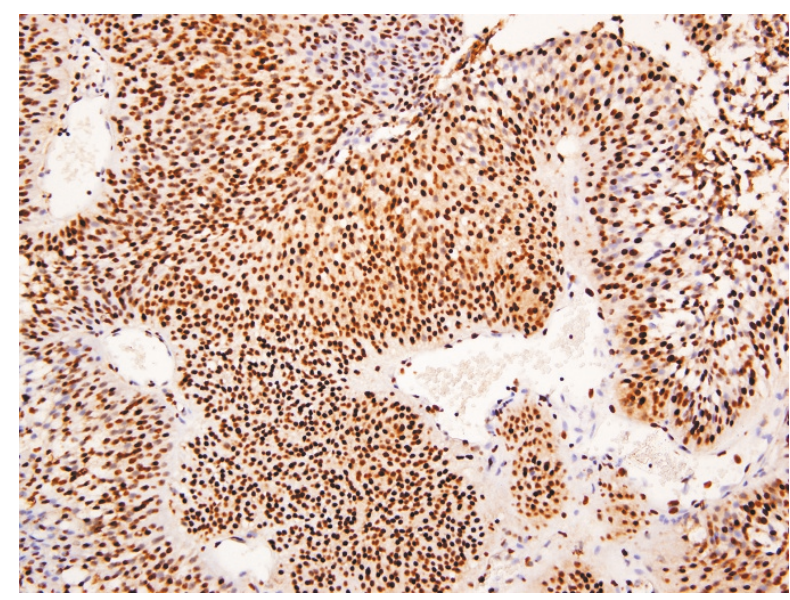

Figure 2. Nucleophosmin/B23 immunostaining of different intensities and percentages was found in the nucleoli, nuclei, and cytoplasm in bladder carcinoma $(\times 100$ magnifications).

nucleophosmin/B23 expression was confined to tumor cells only. For each individual section, the percentage of tumor cell-positive staining ranged from $1 \%$ to $90 \%$; however, nucleophosmin/B23 nuclear staining was noted in all tumor cells (Figures 3, 4). Low staining $(<4 \%$ staining tumor cells) was observed in stage Ta tumor specimens (Figure $3 \mathrm{~A}$ ) and in primary tumors (Figure 4A). On the other hand, high staining was observed in tumors of advanced stages (Figure 3B-3D) and in recurrent tumors (Figure 4B). The overall densitometric analysis of the immunostaining indicated that the upregulated nucleophosmin/B23 protein expression was significantly different between pT2-T4 and pTa, grades $2-3$ and grade 1 as well as between primary and recurrent tumors $(P<0.05 \text {; Figure } 5)^{[9]}$.

Correlation between nucleophosmin/B23 protein 

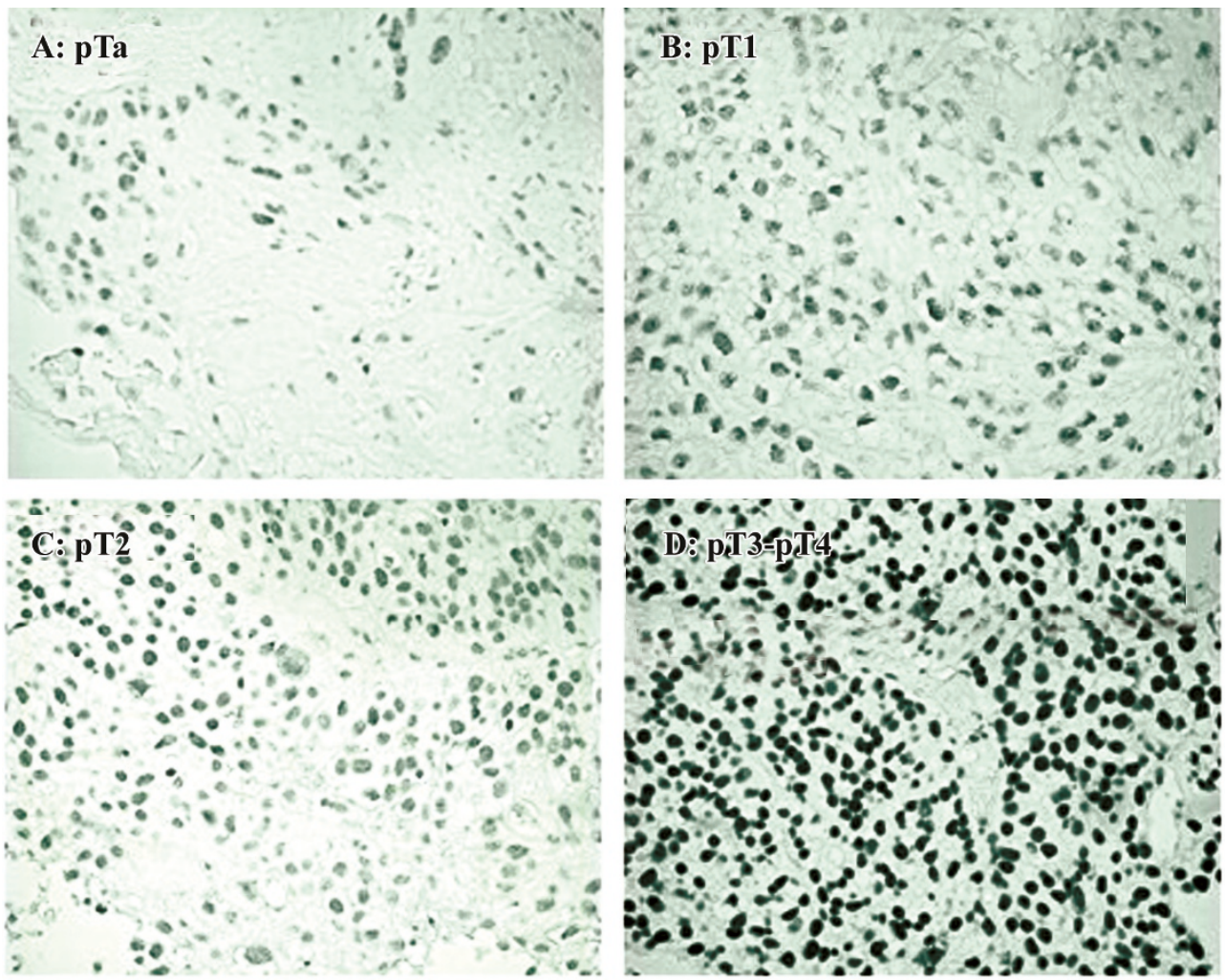

Figure 3. Representative nucleophosmin/B23 immunostained tumor sections (A-D) are TCC $(\times 20$ magnification). Immunostaining of specimen $\mathrm{A}$ (pTa) and B (pT1) shows positive immunoreactivity for nucleophosmin/B23 in a few tumor cells. Strong, positive immunoreactivity for nucleophosmin/B23 in invasive bladder carcinoma is shown in C (pT2) and D (pT3-pT4).
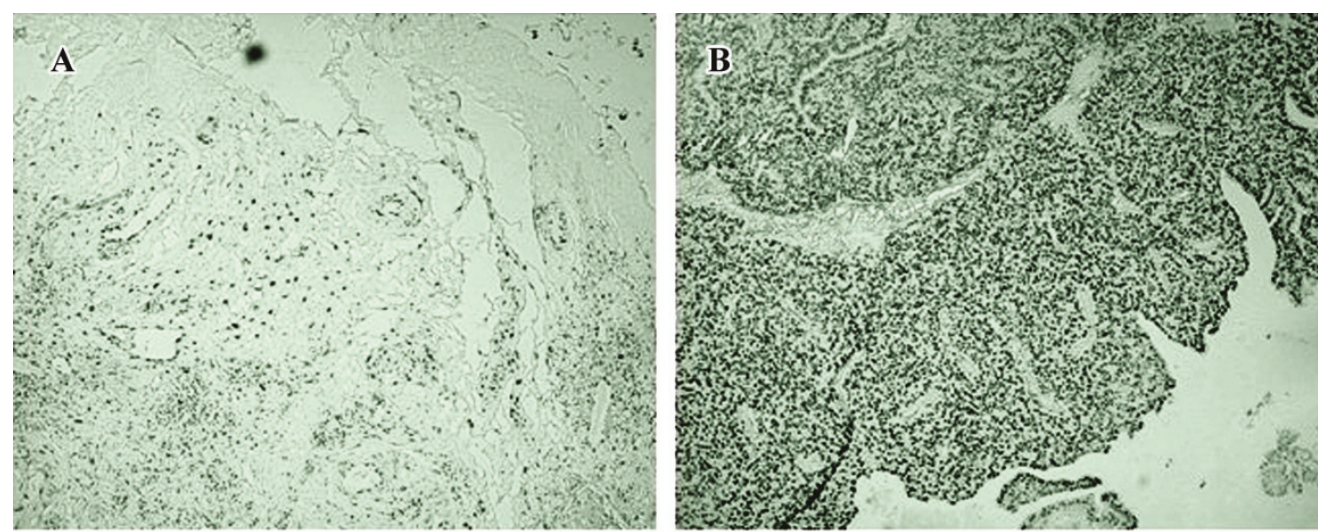

Figure 4. Nucleophosmin/B23 immunohistochemical study of primary and recurrent bladder carcinoma. Expression of nucleophosmin/B23 by immunohistochemical staining in primary bladder tumors (A) or in recurrent tumors (B).

expression and $\mathbf{T}$ classification, tumor grade, and tumor recurrence The percentage of nucleophosmin/B23 staining in pTa tumors was used as a reference value $(<4 \%$ staining tumor cells). The possible association of nucleophosmin/B23 protein levels with clinical and pathological factors in bladder carcinoma was then assessed. Seventeen $(68 \%)$ of 25 pT2-T4 cases had nucleophosmin/B23 staining densitometry values higher than the reference value $(P=0.049$; Table 1). Furthermore, 31 (72.1\%) of 43 cases of grade 1 tumors had low immunostaining densitometry 

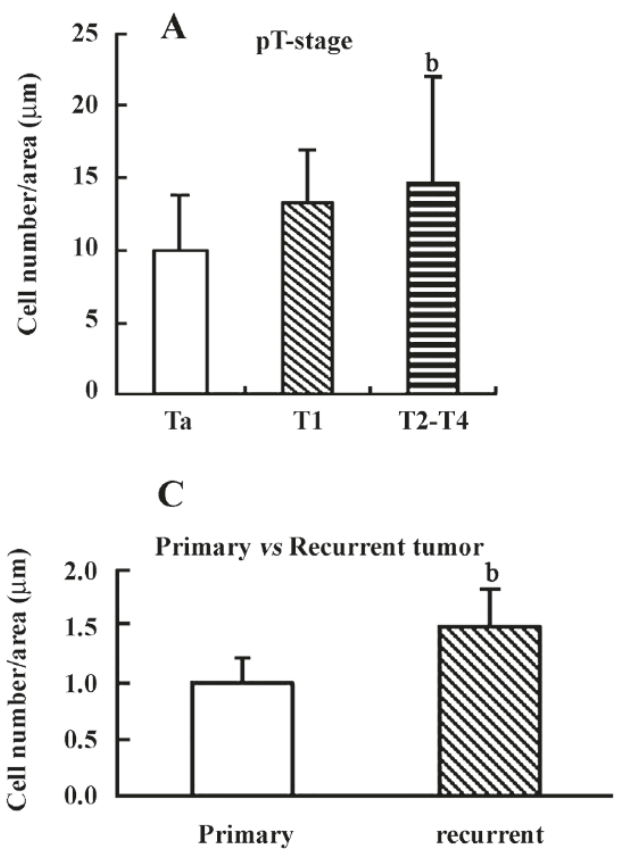

values, whereas $22(61 \%)$ of 36 cases of grade 3 had high immunostaining densitometry values $(P=0.039$; Table 1$)$. Next, the possible relationship between the nucleophosmin/ B23 staining densitometry values and tumor recurrence was also determined. Thirty-five $(60.3 \%)$ of 58 recurrent tumors had high staining values, while $43(51.8 \%)$ of 74 primary tumors had low staining values $(P=0.047$; Table 1$)$. No statistically significant relationship was found between the nucleophosmin/B23 immunostaining densitometry values and age $(P=0.08)$ or sex $(P=0.817$; Table 1$)$.

Upregulated expression of nucleophosmin/B23 is identified as a significant and independent prognostic factor Comparable tumor recurrence was noted in bladder cancer with high and low nucleophosmin/B23 immunostaining densitometry values. In the univariate analysis, high nucleophosmin/B23 staining correlated with a poor prognosis when assessed as a categorical variable greater than the pTa reference value $(P<0.05)$. The Cox multivariate regression analysis with clinicopathological factors identified nucleophosmin/B23 overexpression as the only independent prognostic factor $(P=0.009$; Figure 6$)$. The nucleophosmin/B23 expression correlated significantly with time to bladder carcinoma recurrence ( $P=0.009$; Figure 6$)$.

\section{Discussion}

A major concern of superficial bladder cancers is the relative high recurrence rate. More than a half of superficial tumors recur within 5 years and $10 \%-20 \%$ of these

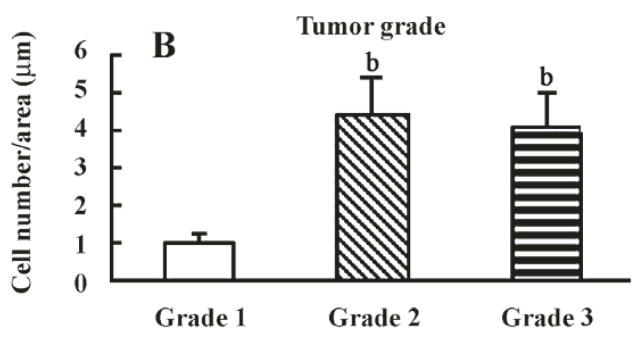

Figure 5. Association of nucleophosmin/B23 immunostaining densitometry values with stages and grades of primary and recurrent tumors. Expression of nucleophosmin/B23 protein levels determined by immunostaining $(\times 20$ magnification) densitometry values of various tumor stages (pTa, pT1, and pT2-pT4), tumor grades (grades 1-3), or primary and recurrent tumors. ${ }^{\mathrm{b}} P<0.05$.

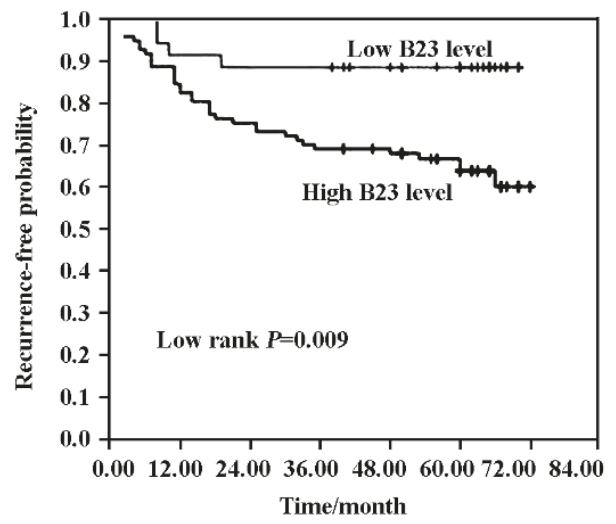

Figure 6. Actuarial 5-year tumor recurrence rates in patients with high or low degrees of nucleophosmin/B23 immunostaining.

progress to invasive disease ${ }^{[10]}$. It is thus important to detect the early events during the recurrence of superficial cancer before the cancer cells undergo changes that lead to invasiveness. Clinical and histopathological factors that might assist in the prediction of tumor recurrence and the progression of bladder cancer have been studied ${ }^{[11]}$. These parameters, when used with the TNM system, will serve as useful and specific tools to provide crucial information about the treatment and prognosis of cancer. An ideal prognostic factor must be reliable, so treatment decisions can be made directly on individuals. Several potential diagnostic markers for bladder cancer have been identified, including nuclear matrix protein 22 , bladder tumor antigen, and 
telomerase ${ }^{[12]}$. Although analyses of these markers allow a sensitive detection of bladder cancer, their use is limited by low specificity. On the other hand, specific genetic alterations have been implicated in the molecular pathogenesis of transitional cell carcinoma, with mutations reported in the cell cycle regulatory genes, oncogenes, and tumor suppressor genes ${ }^{[13]}$. However, it has proven difficult to use these genetic alterations as diagnostic markers of bladder cancer because of their low sensitivity. All these drawbacks in the existing methods have prompted the search for more reliable molecular markers of bladder cancer.

Urothelial carcinoma progression is a multistep process with successive oncogenic changes from the original monoclonal tumorigenic cells. The evaluation of a longterm outcome for superficial bladder cancer has employed actuarial methods ${ }^{[14,15]}$. Furthermore, risk group classification based on prognostic factors could well define progression, mortality, and recurrence rates in primary superficial bladder cancer. A patient with recurrence at 3 months had numerous and frequent recurrences; these tumors also progressed $^{[13]}$. While multiple tumors and recurrence at 3 months were significant prognostic factors for recurrence and progression, nucleophosmin/B23 immunoreactivity significantly correlated with the recurrence rate may be proven clinically useful for the early prediction of bladder cancer progression.

Nucleophosmin/B23 has been known to have a multifunctional role in cells. It has been implicated as an increased nucleolar activity that is necessary for cell proliferation, as a cytoplasmic/nuclear shuttle protein, in relieving transcription repression by YY1, in binding to nuclear and nucleolar localization signals on the HIV type 1 Rev protein and the human T-cell leukemia virus-1-Rex protein, in binding to cell cycle-regulated nucleolar protein p120, and in inhibiting DNA-binding and transcriptional activity of interferon regulatory factor- 1 , which is a tumor suppressor $^{[17-19]}$. Nucleo-phosmin/B23 is significantly more abundant in tumor and proliferating cells than normal resting cells. Nucleophosmin/B23 overexpression at the RNA and protein levels may contribute to the onset of cancer ${ }^{[20]}$. The blockage of the nucleo-phomin/B23 expression with its antisense oligonucleotides has shown that nucleophosmin/ B23 is crucial for rendering cancer cells resistant to induction of differentiation and apoptosis ${ }^{[3,4]}$. It thus appears that an excess of nucleophosmin/B23 may contribute to cancer progression.

A significant association between the nucleophosmin/ B23 protein level and histological subtype was established in this study. The immunohistochemical analysis showed that nucleophosmin/B23 protein staining is importantly associated with tumor stage, histological grade, and recurrence $(P=0.05)$. Tumors with relatively high nucleophos$\mathrm{min} / \mathrm{B} 23$ levels are poorly differentiated at high stage, and have a high recurrence rate. Such a clinical correlation may arise from increased nucleophosmin/B23 activity, which consequently leads to increased cell growth rate and poorly-differentiated tumor phenotypes. This result also indicates a potentially important role of nucleophosmin/B23 in the development and progression of bladder carcinoma. The mechanism by which nucleophosmin/B23 overexpression is involved in cancer recurrence and progression is not clear at present and requires further investigation.

In summary, the Cox multivariate analysis has implicated that the overexpression of the nucleophosmin/B23 protein, assessed by immunohistochemistry, is independently associated with bladder cancer recurrence. Upregulated nucleophosmin/ B23 protein expression can thus serve as an independent and effective prognostic factor for bladder cancer. The high percentage of tumors with positive staining of nucleophosmin/B23 in this study confirms its crucial role in the pathogenesis of bladder carcinoma. The discovery of nucleophosmin/B23 as a key target in bladder cancer recurrence and progression will soon permit better diagnosis and therapeutic intervention.

\section{Acknowledgements}

We thank Dr Bertrand Chin-ming TAN for carefully proofreading the manuscript

\section{References}

1 Partin DM, Bray F, Ferlay J, Pisani P. Global cancer statistics. CA Cancer J Clin 2005; 55: 74-108.

2 Heney N, Ahmed S, Flanagan MJ, Frable W, Corder MP, Hafermann $\mathrm{MD}$, et al. Superficial bladder: progression and recurrence. J Urol 1983; 130: 1983-6.

3 Hsu ZY, Yung BYM. Down-regulation of nucleophosmin/B23 during retinoic acid-induced differentiation of human promyelocytic leukemia HL-60 cells. Oncogene 1998; 16: 915-23.

4 Liu WH, Yung BYM. Mortalization of human promyelocytic leukemia HL-60 cells to be more susceptible to sodium butyrate-induced apoptosis and inhibition of telomerase activity by down-regulation of nucleophosmin/B23. Oncogene 1998; 17: 3055-64.

5 Feuerstein N, Spiegel S, Mond JJ. The nuclear matrix protein, numatrin (B23), is associated with growth factor induced mitogenesis in Swiss $3 \mathrm{~T} 3$ fibroblasts and with T lymphocyte proliferation stimulated by lectins and anti-T cell antigen receptor antibody. J Cell Biol 1988; 107: $1629-42$.

6 Tsui KH, Cheng AJ, Chang PL, Pan TL, Yung BYM. Association of 
nucleophosmin/B23 mRNA expression with clinical outcome in patients with bladder carcinoma. Urology 2004; 64: 839-44.

7 Sobin LH, Wittekind CH. Urinary bladder. In Sobin LH, editor. TNM classification of malignancy tumors, v5. 5th ed. New York: Wiley-Liss; 1977. p 187-9.

8 Mostofi FK, Sobin LH, Torloni H. Histological typing of urinary bladder tumors. In: Mostofi FK, editor. International classification of tumors, No. 10. Geneva: World Health Organization, 1973.

9 Suash S, Mehar CS, Deepak KG, Chitra S. Angiogenic patterns and their quantitation in high grade astrocytic tumors. J Neurooncol 2006; 79: 19-30.

10 Raghaven D, Shipley WU, Garnick MB, Rusell PJ, Richie JP. Biology and management of bladder cancer. N Engl J Med 1990; 322: 1129-38.

11 Ramakumar S, Bhuiyan J, Besse JA, Rusell PJ, Richie JP. Comparison of screening methods in the detection of bladder cancer. J Urol 1999; 161: 388-94.

12 Tsui KH, Chen SM, Wang TM, Juang HH, Chen CL, Sun GH, et al. Comparison of voided urine cytology, nuclear matrix protein-22 and bladder tumor associated antigen tests for bladder cancer of geriatric male patients in Taiwan, China. Asian J Androl 2007; 9: 711-5.

13 Tada Y, Wada M, Taguchi K, Mochida Y, Kinugawa N, Tsuneyoshi M. The association of death-associated protein kinase hypermethylation with early recurrence in superficial bladder cancers. Cancer Res 2002; 62: 4048-53.

14 Sidransky D, Von Eschenbach A, Tsai YC, Jones P, Summerhayes I,
Marshall F. Identification of p53 gene mutations in bladder cancers and urine samples. Science 1991; 252: 706-9.

15 Parmar MK, Freedman LS, Hargreave TB, Tolley DA. Prognostic factors for recurrence and followup polices in the treatment of superficial bladder cancer: report from the British Medical Research Council Subgroup on Superficial Bladder Cancer (Urological Cancer Working Party). J Urol 1989; 142 (2 Pt 1): 284-8.

16 Morris SB, Gordon EM, Shearer RJ, Woodhouse CR. Superficial bladder cancer: for how long should a tumor-free patient have check cystoscopies? Br J Urol 1995; 75: 193-6.

17 Millan-Rodriguez F, Chechile-Toniolo G, Salvador-Bayarri J, Palou J, Algaba F. Primary superficial bladder cancer risk groups according to progression, mortality and recurrence. J Urol 2000; 164: 680-4.

18 Inouye CJ, Seto E. Relief of YY1-induced transcriptional repression by protein-protein interaction with the nucleolar phosphoprotein B23. J Biol Chem 1994; 269: 6506-10.

19 Valdez BC, Perlaky L, Henning D, Saijo Y, Chan PK, Busch H. Identification of the nuclear and nucleolar localization signals of the protein p120: interaction with translocation protein B23. J Biol Chem 1994; 269: 23 776-83.

20 Kondo T, Minamino N, Nagamura-Inoue T, Matsumoto M, Taniguchi T, Tanaka N. Identification and characterization of nucleophosmin/ B23/numatrin which binds the anti-oncogenic transcription factor IRF-1 and manifests oncogenic activity. Oncogene 1997; 15: 1275 81. 